\title{
FACTORES SOCIALES EN LAS CONDUCTAS ALIMENTARIAS DE ESTUDIANTES UNIVERSITARIOS
}

\section{SOCIAL FACTORS IN FEEDING BEHAVIOR OF UNIVERSITY STUDENTS}

\author{
Claudia Troncoso P., Juan Pablo Amaya P. \\ Departamento de Clínica y Preclínica. Facultad de Medicina. \\ Carrera de Nutrición y Dietética. Universidad Católica de la Santísima Concepción, Concepción, Chile.
}

\begin{abstract}
The feeding behavior has a direct influence on the nutritional status of individuals belonging to diverse age groups, among them, higher education students. The aim of this research was to interpret the perception of the influence exerted by the family environment, university peers and educational staff on the dietary behavior of university students belonging to the Universidad Católica de la Santísima Concepción. In order to achieve this aim, qualitative methodology through a phenomenological design was used. As a strategy to gather information, a semi-structured interview was applied to 8 students, going into some of the results in depth with a focus group composed by 9 students of the university. Our results showed that the family, friends and university staff influenced the feeding behavior of the interviewed students. Moreover, these influences were found to be favorable regarding healthy feeding behavior in the case of studentis family; and unfavorable in the case of friends and university staff. Key words: University students, feeding behavior, social factors, qualitative study.
\end{abstract}

Este trabajo fue recibido el 4 de Mayo de 2009 y aceptado para ser publicado el 20 de Septiembre de 2009.

\section{INTRODUCCIÓN}

El ser humano, como todo organismo vivo, se comporta como un sistema complejo e integrado, que presenta la capacidad de generar y mantener sus propias estructuras y permitir un equilibrio interno constante. Puede, además, relacionarse con el medio que le rodea y lograr metas específicas, a través de la autoprogramación, autorregulación y la adaptación. Estas últimas son propiedades que corresponden a la génesis del comportamiento humano (1). Se puede definir conducta como cualquier proceso o actividad humana o animal, que puede observarse o medirse objetivamente y con neutralidad (sin influencias de juicios de valores, preferencias personales o prejuicios y con el acuerdo de distintos observadores), que tienen como objetivo el cubrir una determinada situación carencial en las personas (2-3).

Entre las conductas adquiridas por los seres humanos, las alimentarias pueden ser definidas como un comportamiento habitual relacionado con hábitos alimentarios, selección de alimentos, preparaciones y volúmenes consumidos de estos alimentos, los que influyen directamente en el estado nutricional de los individuos pertenecientes a diversos grupos etarios (4). Es un fenómeno de naturaleza compleja y de origen multifactorial, que por definición es influenciado por diversos factores entre los cuales se distinguen la sociedad, cultura, religión, situación económica, disponibilidad de alimentos entre otros (5-6). La regulación de la alimentación es la resultante de una serie de procesos que incluyen señales hormonales, metabólicas y neuronales, los que son integrados en el hipotálamo, órgano encargado de generar respuestas de cesación o de búsqueda de alimentos (7).

Los hábitos alimentarios se adquieren desde la infancia y estas pautas alimentarias pueden ser el origen de patologías crónicas relacionadas con la malnutrición por exceso en edades posteriores (8). La Organización Panamericana de la Salud en su Estrategia Regional y Plan de Acción para un Enfoque Integrado Sobre la Prevención y Control de las Enfermedades Crónicas del año 2007 (9), reconoce factores ambientales, económicos 
y sociales y sus consiguientes procesos conductuales y biológicos que actúan en los diversos ciclos de la vida, que inciden sobre el riesgo de presentar algún tipo de patología crónica. En los adultos estos riesgos se refieren a la ingesta excesiva de grasas de tipo saturada y de sal, el bajo consumo de frutas y verduras, además del consumo de tabaco, alcohol, baja actividad física y factores de riesgo biológicos asociados. En el año 2006 el Ministerio de Salud de Chile realizó la segunda Encuesta de Calidad de Vida (10), que entre otras variables medidas, determinó algunos hábitos alimentarios en la población chilena entre los 15 a 75 años o más, según sexo y nivel socioeconómico al que pertenecían. Entre sus resultados se concluyó, por ejemplo, que $74.7 \%$ de la población presenta entre sus conductas alimentarias el consumo de desayuno en forma diaria (pregunta 43 ) o que $52.4 \%$ de la población estudiada refiere nunca agregar sal a las comidas en la mesa (pregunta 44).

Los estudiantes universitarios corresponden a una población adulta joven clave para las actividades de promoción y prevención en salud. A partir del año 2006, el Ministerio de Salud de Chile tiene entre sus políticas públicas la "Guía para Universidades Saludables y otras Instituciones de Educación Superior" (11) destinada a estudiantes, académicos y personal de apoyo de la educación superior, que tiene como meta su implementación para mejorar su calidad de vida y salud en este grupo objetivo. Entre las recomendaciones que entrega esta guía para los casinos y confiterías que se encuentren en los establecimientos educacionales está la utilización adecuada de las becas de alimentación de los estudiantes y los bonos de alimentación de los funcionarios; presencia de salad-bar en base a lo menos tres variedades de ensaladas crudas y cocidas; oferta de pescados o leguminosas a lo menos 1 ó 2 veces a la semana; privilegiar las preparaciones al horno, al vapor, a la plancha o cocidas por sobre las fritas; postres con dos alternativas de frutas naturales o preparadas y postre elaborado, con consideraciones nutricionales o de aceptabilidad.

Estadísticas realizadas por el Ministerio de Educación de Chile (12), indican que para el año 2007, existía una matrícula de 726.285 estudiantes de pre-grado de instituciones universitarias, institutos profesionales y centros de formación técnica. De estos, 482.265 pertenecían a universidades tradicionales o privadas.

Frente al perfil epidemiológico de la población adulta del país y a la importancia que presentan los estudiantes universitarios como grupo estratégico para la promoción en salud, el objetivo de esta investigación fue interpretar la percepción de los estudiantes universitarios de las condicionantes que ejercen la familia, entorno social y plantel de educación superior en sus conductas alimentarias.

\section{SUJETOS Y MÉTODOS}

El diseño cualitativo de tipo fenomenológico fue utilizado para el desarrollo de esta investigación, realizada con estudiantes pertenecientes a la Universidad Católica de la Santísima Concepción, ubicada en la región del Bío-Bío, Chile.

Para ser seleccionados en el estudio los participantes debían ser alumnos regulares de pregrado de alguna carrera impartida en el Campus San Andrés de la Institución de Educación Superior antes mencionada, con a lo menos un año de permanencia en el establecimiento, mayor de 18 años y que participaran en forma voluntaria en el estudio, firmando consentimiento informado.

La estrategia para la obtención de la muestra correspondió a un muestreo a base de criterios, utilizando para la selección de individuos los criterios antes mencionados. El cálculo de la muestra resulta del punto de saturación, es decir, considerando que este se produce cuando el $50 \%$ de las respuestas entregadas comienzan a repetirse.

Las estrategias utilizadas para la obtención de información correspondieron a entrevistas individuales y grupo focal. El tipo de entrevista individual realizada fue semi-estructurada, desarrollada por uno de los investigadores. Las preguntas aplicadas se basaban en el desarrollo del discurso en donde se genera la percepción de estos estudiantes sobre de las influencias de su familia, amigos de la universidad y establecimiento de educación superior en sus conductas alimentarias. Las entrevistas fueron realizadas en condiciones estándar en el establecimiento universitario; grabadas y traspasada a formato papel para ser posteriormente analizadas por investigador principal. El tiempo destinado a la aplicación de las entrevistas correspondió a 60 minutos en promedio (r: 40-80 minutos). El grupo focal fue utilizado para profundizar algunos resultados encontrados en las entrevistas, referidos especialmente a la influencia de la familia y la universidad en las conductas alimentarias. La metodología para la obtención de la información se realizó en condiciones similares a las entrevistas, con un grupo conformado por nueve estudiantes que cumplían con los criterios de selección del estudio y que firmaron el consentimiento respectivo de participación. El tiempo utilizado para la realización del grupo focal fue 40 minutos.

El punto de saturación para las entrevista correspondió a ocho estudiantes. Los resultados de las entrevistas y grupo focal se triangularon y analizaron a través del análisis semántico estructural. 


\section{RESULTADOS}

\section{Percepción de sus conductas alimentarias}

El concepto "conducta alimentaria" para gran parte de los entrevistados corresponde a la forma en que una persona se alimenta o debe alimentarse. “...Visiones que tiene uno que es lo que debería y lo que no debería comer..." [Entrevista 8]. Para uno de los entrevistados, conducta alimentaria corresponde a las costumbres que presentan los individuos al alimentarse, originado en la familia. Estas costumbres, las codifica como la transmisión de padres a hijos y que se acrecienta al ser partícipe de la sociedad. "A costumbres como los padres enseñan la alimentación que les van proporcionando, la alimentación que se come en el hogar y que uno va después adquiriendo con los estudios con toda la sociedad" [Entrevista 7].

Existe un consenso entre los estudiantes entrevistados al percibir sus conductas alimentarias como inadecuadas. Los motivos señalados por estos últimos para llegar a esta conclusión se basan especialmente en la falta de disponibilidad de tiempo para cumplir con horarios de alimentación en el plantel universitario o a la falta de hábitos en la incorporación de horarios de alimentación, lo que impediría mantener conductas alimentarias adecuadas. “...A veces aquí es difícil estar en la U, estar estudiando, llevar una conducta, una dieta balanceada porque a veces no hay tiempo de almorzar...no sé...no tomo desayuno. A veces me quedo con el desayuno y en el día me como no sé un pan y unas papas fritas y en la noche llego a almorzar a mi casa, a comer, entonces no hay como un orden, no hay un orden de alimentación" [Entrevista 6]. "....No me dedico en pensar lo que uno come, si no que principalmente uno come a deshoras..." [Entrevista 2]. “...Porque nunca he tenido hábitos alimentarios adecuados, no tomo desayuno, a veces no tomo once y a veces tomo todo junto" [Entrevista 7]. Sólo dos estudiantes definen sus conductas alimentarias como adecuadas, basándose en la calidad de los alimentos que consumen o en la incorporación de alimentos considerados como sanos para el entrevistado. “...Más sana; para empezar más equilibrada, mayor variedad de productos que consumo en el día..." [Entrevista 4]. "Yo considero que no esta tan mal porque hay otros que comen peor que yo y nunca como cosas así como comida chatarra, o sea, soy así como bien mala para esas cosas, siempre para mí es más importante un plato de comida" [Entrevista 1].

Para algunos de los entrevistados los únicos horarios de alimentación en que las comidas pueden ser catalogadas como "saludables" son las consumidas en el establecimiento universitario (refiriéndose a la alimentación entregada en el Casino del plantel). Esto se debe a que algunos estudiante codifican que en sus hogares deben ingerir los alimentos que estén disponibles: “...Porque yo creo que lo único saludable que como es acá en la U, y en la casa yo como lo que hay" [Entrevista 1]. "Igual acá en la universidad tratan de hacer un menú nutritivo, en la ley por decirlo de alguna forma. Pero nosotros nos vamos al otro extremo los días o no tenemos clase o estamos en la casa y hasta ahí queda como el orden de la alimentación., porque estamos en la casa y tenemos mantequilla, cecinas, queso todo tipo de cosa que nos engordan..." [Entrevista 8].

\section{Percepción de las influencias que condicionan sus conductas alimentarias}

Para la mayoría de los estudiantes universitarios entrevistados, sus conductas alimentarias en su etapa académica se perciben condicionadas a su estado anímico o a situaciones de estrés. “...Para mí a veces mi estado anímico, emocional, afecta mi alimentación, sin duda" [Entrevista 4]. Estas modificaciones se basan en la incorporación de alimentos que aportan hidratos de carbono de absorción rápida o también el consumo de café. "Cuando hay certámenes o estoy muy estresada me da como más hambre o comer chocolates o esas cosas" [Entrevista 6]. Uno de los entrevistados menciona que esta modificación alimentaria es afectada por la disponibilidad de alimentos en su hogar, pero que son sus preferencias personales las que finalmente condicionará la selección de alimentos. “...Lo primero que siempre encuentra y siempre va a haber es pan, pan o juguito heladito, es difícil encontrar siempre una fruta, o sea si voy a elegir entre un pan o una fruta casi siempre voy a elegir el pan con algo es como más sabroso, más rico...". [Entrevista 4].

Algunos de los estudiantes perciben que la publicidad influencia en sus conductas alimentarias, aunque no es suficiente como para modificarlas. “...O sea yo no es que me influencie, pero si eh... hoy en día cada comercial o en cada intertanto comercial mínimo aparecen dos o tres productos eh... alimenticios o saludables, pero más que nada lo tomo como informativo, no como que voy a ir a comprar ese producto, no" [Entrevista 2]. Uno de los entrevistados le da una connotación positiva a la publicidad en influencias de las conductas alimentarias, pero destinada a la situación actual de salud a la población en general. “...Le dan harto énfasis al consumo de verduras o hacen propaganda de buena salud, de hacer ejercicios entonces yo creo que están tratando de cambiar....". Yo creo que están tratando de cambiar en ese sentido por las tasas yo creo de obesidad o de problemas cardiovasculares que hay, yo creo que están tratando de dar vuelta 
ese problema". [Entrevista 6]. "Las propagandas, porque hay algunas que son demasiadas llamativas y eso igual como que tiende a conducir a la persona a comprar esa cosas, a comprar algo" [Entrevista 8].

Una de las percepciones que influencian las conductas alimentarias en parte de los entrevistados es el agrado que ciertos alimentos conllevan o la voluntad que existe en la selección de estos últimos, sin considerar en algunos casos sus características nutricionales. "No, como siempre lo mismo, me preocupo que me guste no más, no en sabores o modas o porque me dijeron que era rico, sólo que me guste” [Entrevista 2]; “... A mi me gustan las cosas ricas, eso es lo que como, para mi lo más rico son las verduras, prefiero un plato de verdura en vez de un plato de arroz con carne" [Entrevista 1].

Para la mayoría de los entrevistados, el tiempo disponible es un factor importante en sus conductas alimentarias. La falta de tiempo condicionaría la forma de alimentarse en algunos alumnos del plantel de educación superior estudiado. "El estudio... que no puedo, que no me dedico porque realmente sino tengo tiempo no como, no más paso de largo y cuando llego a la casa como cualquier cosa que pille..." [Entrevista 7].

\section{Percepción del entorno familiar en sus conductas alimentarias}

Para todos los entrevistados, existen influencias por parte de la familia en sus conductas alimentarias. Estas, para la mayoría, son consideradas como positivas en la forma de alimentarse.

$\mathrm{Al}$ identificar un integrante del grupo familiar que influya en la manera de alimentarse, gran parte de los estudiantes mencionan a su madre. Para algunos de estos, al ser ella quien prepara los alimentos, es quien finalmente condiciona el mantener o realizar una alimentación saludable. Esta influencia y la etiqueta del rol de la madre son corroboradas por los estudiantes que participaron en el grupo focal. “...Creo porque en mi casa mi mamá hace como puras comidas sanas, es como que ella se cuida las comidas en ese sentido, de comer legumbres, verduras evita, casi nunca, rara vez hace frituras" [Entrevista 6]; “...Siempre tratando de fomentar el consumo de frutas, de verduras, por ejemplo. Cuando cocina trata de hacer que la fruta y la verdura, que generalmente no es algo tan atractivo que se vuelva tan atractivo, o por ultimo que pase "piola" por decirlo de algún modo, entre medio de otras cosas más agradables" [Entrevista 5]. Parte de los entrevistados refiere que su madre, en el rol protector que realiza, influye en su forma de alimentarse y en instar a presentar estilos de vida más saludables. “...Entonces ella siempre esta pendiente: "Hijo no coma tanto, haga más ejercicio, tratemos de variar las cosas..." [Entre- vista 4]. "...Más bien por eso ella es preocupada de la alimentación, siempre uno anda preocupado de eso..." [Entrevista 2].

El padre también es percibido por algunos estudiantes como una persona, que en su grupo familiar, influye en la alimentación. Sin embargo, esta influencia está dada por el rol de proveedor que este representa para algunos entrevistados. Uno de los últimos menciona que al ser él quien adquiere los alimentos para el hogar, impide que su alimentación y la de su grupo familiar sea variada, lo que repercutirá en su estado de salud: “...y siempre trataba de mantener un equilibrio en cuanto al consumo de dinero y por ende no variaba mucho el menú de la semana y del día y entonces eso igual repercutía en la alimentación de él, de mi mamá y la mía....". [Entrevista 4].

Varios de los entrevistados mencionan que la situación económica que presenta su grupo familiar influye en su forma de alimentarse, favoreciendo o perjudicando la calidad alimentaria que estos presentan. "Eh... no sé, creo que sí. Es que también tiene que ver ahí un factor socioeconómico, porque si no hay plata para comprar cosas saludables no se pueden comer cosas saludables..." [Entrevista 1].

Uno de los entrevistados, menciona que la forma de alimentarse en su núcleo familiar, es condicionada por algunas patologías que presentan sus progenitores, por lo que su alimentación en el hogar va a depender de restricciones alimentarias que estos tienen. “...Por lo mismo uno para evitar conflictos uno trata de consumir cosas que no le hagan mal a ellos para evitar los conflictos" [Entrevista 2].

\section{Percepción del entorno social en sus conductas alimentarias}

La mayoría de los entrevistados perciben como entorno social a sus pares en la universidad. Para gran parte de los estudiantes existirían influencias en sus conductas alimentarias, dada principalmente en la instancia de selección de alimentos. "Yo creo que mis compañeras más que mis compañeros, porque mis compañeras tienden más a cuidarse. Por ejemplo, en el Casino ofrecen el hipocalórico y la opción más tradicional, ellas van con el hipocalórico; en el Mall a pesar que es comida chatarra tratan de elegir lo más liviano, "entre paréntesis". En cambio mis compañeros me dicen: "elige algo que te llene", entonces empiezan compra esto, esto otro, porque llena más". Entonces, esa es la diferencia" [Entrevista 8].

Algunos de los entrevistados mencionan que prefieren consumir una alimentación no adecuada para ellos que alimentarse sin la compañía de sus pares univer- 
sitarios. “...Si yo voy al casino y por no comer solo, de repente puedo comer algo que no me parezca sano, porque el almuerzo que esta ahí no le gusta a nadie de mi grupo por ejemplo, y nos vamos a otro lado a comer quizás algo no tan sano, no tan preparado pero para no comer solo". Sin embargo, esta influencia es sólo circunstancial: "....de repente por no comer solo se termina comiendo lo que come el grupo" [Entrevista 5]. Para uno de los entrevistados es una instancia de esparcimiento con sus compañeros; un momento de socialización y de consumir alimentos que le son placenteros: “... Obvio yo no le voy a decir que no, para que poh, es una instancia en que vamos a salir un poco de la vida de la Universidad, de la rutina de la Universidad, y vamos a poder compartir, reír un poco y comer algo rico..." [Entrevista 4].

\section{Percepción del entorno universitario en sus conductas alimentarias}

La mayoría de los entrevistados perciben que la Institución Universitaria influye en sus conductas alimentarias. Alguna de las respuestas entregadas por los estudiantes menciona la falta de información para mantener o realizar una alimentación saludable. “...Desde que entré a la universidad, yo no he visto que venga una nutricionista a hacernos una charla como curso: "chiquillos les aconsejo que se alimenten cada tantas horas, que pongan mas énfasis en estos productos, en estos alimentos..." [Entrevista 4].

La falta de disponibilidad de tiempo para realizar los horarios de alimentación en el establecimiento educacional también es mencionada por la mayoría de los entrevistados. Se percibe que esta carencia impide el desarrollo de adecuadas conductas alimentarias, referido especialmente al horario de almuerzo. "Porque no da el tiempo a veces. Porque en el casino a veces hay la media cola, hasta el gimnasio esta la cola para esperar a almorzar y salgo 12.40 y en hacer la cola llego a las 13.30 y entro a clases a las 14.00 hrs. ó 13.45 llego a sacar recién la bandeja y no alcanzo a almorzar. No se da el tiempo" [Entrevista 6]. Algunos de los estudiantes reconocen política instaurada por la Institución, entregando una franja horaria para colación, sin embargo, esta no es suficiente para lograr realizar este horario de alimentación, lo que se traduce en algunos casos en no consumir adecuadamente su almuerzo o la inasistencia a sus actividades académicas. "Igual en algunos casos es súper corta porque o no sé si es un problema de espacio, porque el casino a la larga se hace chico porque colas que van desde el mismo casino al gimnasio a veces. No sé, yo pienso a veces que el horario podría ser más extenso para comer más tranquilo porque uno tiene que estar comiendo rápido porque o tiene la hora que entra a clase y al final afecta porque uno llega tarde a clase. Siempre por lo general trato de comer, no me queda otra que comer rápido porque si no llego a clases o... en realidad no llego a clases" [Entrevista 8]. Esta falta de disponibilidad horaria en el quehacer universitario es mencionada por los estudiantes que participaron en el grupo focal.

Es interesante la percepción que algunos de los estudiantes presentan del hecho de recibir alimentación en el establecimiento universitario, específicamente en el horario de almuerzo. “... Yo he visto que está bien equilibrada la alimentación, un muy buen menú todos los días, porque me he encontrado con la sorpresa que tiene un plato hipocalórico todos los días, y eso esta bien, por ese lado la Universidad creo que está apoyando una buena alimentación..." [Entrevista 4]. Para dos de los entrevistados, el hecho de poseer beneficio de alimentación permite presentar conductas alimentarias adecuadas, facilitando la selección de alimentos y el recibir una alimentación más variada. “...Porque yo creo que lo único saludable que como es acá en la U, y en la casa yo como lo que hay" [Entrevista 1].

\section{DISCUSIÓN}

Las investigaciones realizadas en estudiantes universitarios se centran especialmente en conductas de riesgo para estos últimos, en las que se incluyen, por ejemplo, la calidad de los alimentos consumidos o el estado nutricional, el uso de drogas lícitas, entre otros factores (13-19).

Entre los resultados obtenidos de investigaciones realizadas en este grupo clave, se destaca la presencia de hábitos alimentarios irregulares, especialmente en los grupos con mal nutrición, ya sea por déficit o por exceso (20). La carrera que estudien no influiría en mantener conductas alimentarias saludables (21), alejándose estas últimas de las actuales recomendaciones dietéticas (22-28).

En los estudiantes entrevistados existiría un consenso en identificar que la forma en que se alimentan no es la más adecuada, situación que se debería especialmente a la limitada disponibilidad de tiempo que la vida universitaria conlleva, ya sea por los horarios o tiempos destinados a las actividades académicas que deben realizar o por las situaciones de estrés que limitan o modifican su alimentación. Se menciona, entre algunas conductas alimentarias inadecuadas, el no realizar algunos horarios de alimentación, como lo son el desayuno y el almuerzo o el consumo de alimentos con alta densidad energética.

Es importante señalar que para algunos de los entrevistados los beneficios estudiantiles que se entregan, 
específicamente una beca alimenticia, son percibidas como positivas y necesarias para el condicionamiento del consumo de una alimentación más saludable o equilibrada, aunque sólo corresponda al horario de almuerzo.

Las situaciones de estrés académico se interpretan como un factor que influye en la modificación de la alimentación de los estudiantes entrevistados, aumentando en algunos estudiantes la ingesta de H. de C. de absorción rápida. Esta situación se ha reportado en este grupo etario en otras universidades de la zona (29).

La familia influiría en la forma de alimentarse en estos estudiantes, interpretando mayoritariamente como positiva la enseñanza entregada por su núcleo parental. En nuestro estudio se identifica, de acuerdo a repuestas de alumnos de ambos sexos, a la madre en su rol social de ser la encargada de "preparar los alimentos", la que favorecería o condicionaría una alimentación saludable. El padre también es percibido por algunos estudiantes, en un rol distinto a la madre: es etiquetado como "proveedor", favoreciendo una alimentación saludable al adquirir alimentos con estas características. La situación económica de la familia es mencionada como condicionante de una alimentación adecuada para el estudiante, limitando la selección de alimentos a los ingresos económicos y por consecuencia, la imposibilidad de mantener conductas alimentarias saludables cuando estos últimos no son favorables.

El entorno social universitario, para la mayoría de los entrevistados, es interpretado como la relación de amistad presente o que surge con los pares de estudios en la universidad, los que influirían en el comportamiento alimentario de estos últimos. Esta influencia se concentraría en la selección de alimentos, la que es supeditada a la compañía de sus amigos en la Universidad, limitando la calidad nutricional de los alimentos que se consumen por la instancia de socialización con los compañeros de estudios. Algunos de los entrevistados mencionan que prefieren ingerir alimentos que codifican como "no saludables" (conociendo o considerando las posibles complicaciones en su estado de salud o calidad de vida), evitando de esta forma alimentarse en forma aislada y favoreciendo la socialización con sus compañeros de universidad.

La publicidad también es mencionada como influyente en las conductas alimentarias de los estudiantes entrevistados, aunque no es suficiente para modificar los hábitos de alimentación.

El plantel universitario también influenciaría la manera de alimentarse de los estudiantes entrevistados, aunque la interpretación que entregan no es mayormente positiva hacia la Institución. Los horarios de la jornada académica son mencionados como un factor negativo para una adecuada alimentación en el plantel. Además, se identifica como insuficiente el tiempo destinado para la colación o almuerzo de los estudiantes, aunque se reconocen políticas internas destinadas a favorecer el consumo de alimentos en este horario de alimentación. Algunos de los entrevistados codifican que la alimentación recibida en el casino universitario cubre con las necesidades nutritivas que requieren, lo que favorecería el mantener una alimentación más "saludable".

\section{CONCLUSIONES}

La interpretación de la percepción de los estudiantes universitarios que participaron en este estudio permite concluir que existen influencias de sus familias, amigos y el plantel universitario en sus conductas alimentarias, los que actúan en forma positiva o negativa en su alimentación.

La familia se interpreta como una institución tradicional formadora del comportamiento alimentario para estos estudiantes, etiquetando roles explícitos de los padres que favorecerían o influirían en desarrollar o mantener una alimentación saludable. Las amistades con pares universitarios y el plantel institucional son identificados negativamente como factores que condicionan el comportamiento alimentario de un número importante de entrevistados. Las amistades influirían en la selección de alimentos, consumiendo algunos de estos últimos identificados como de "no saludables", pero que permite formar o asentar redes sociales con sus pares. Por otra parte, la universidad con su jornada académica (referido a los horarios de clases) es percibida como limitante en el mantener o poder desarrollar conductas alimentarias saludables.

Un hallazgo de esta investigación fue la percepción que presenta un grupo de estudiantes entrevistados al hecho de ser poseedor de un beneficio económico, a través de una beca de alimentación, como un factor condicionante para realizar una alimentación saludable. Esta concepción de lo que significa el poder desarrollar una alimentación saludable nos da una alerta a la importancia que presenta para los planteles universitarios la educación en alimentación y estilos de vida saludables.

El diseño cualitativo presenta como elementos básicos el análisis y la interpretación de las ideas y las palabras de los sujetos entrevistados (30). Los resultados o hallazgos encontrados permiten realizar inferencias lógicas sobre situaciones o poblaciones que sean muy similares a la que se investigó (31). Este tipo de diseño metodológico se presenta como una forma de enriquecer la investigación en salud, utilizando la perspectiva de la persona que se está estudiando.

Por lo mismo, es necesario seguir estudiando desde 
la visión de la persona las condicionantes que favorecen o modifican las conductas alimentarias y la influencia que factores externos o inherentes al individuo influyen en la manera de alimentarse. Esto podría permitir mejorar la acertividad de los programas de promoción y prevención en salud existentes, permitiendo la modificación de conductas en alimentación, favoreciendo la formación de estilos de vida saludables en estos adultos jóvenes.

\section{RESUMEN}

Las conductas alimentarias influyen directamente en el estado nutricional de los individuos pertenecientes a diversos grupos etarios, entre ellos, los estudiantes de educación superior. El objetivo de esta investigación fue interpretar la percepción de los estudiantes universitarios pertenecientes a la Universidad Católica de la Santísima Concepción, de las condicionantes que el entorno familiar, social y plantel educacional ejercen en sus conductas alimentarias. Para el logro de este fin se utilizó la metodología cualitativa, a través de un diseño fenomenológico. Como estrategia de recolección de información se realizó una entrevista semi-estructurada a 8 estudiantes, profundizando algunos resultados con un grupo focal integrado por 9 alumnos de la Universidad. Por los resultados y en conclusión del estudio, la familia, amigos y plantel universitario condicionan las conductas alimentarias de los estudiantes entrevistados, interpretando estas influencias como favorables en el caso de la familia y desfavorables por parte del plantel universitario y los amigos de aulas para mantener conductas alimentarias saludables.

Palabras clave: Estudiantes universitarios; conductas alimentarias; factores sociales; estudio cualitativo.

Dirigir la correspondencia a:

Nutricionista

Claudia Troncoso Pantoja

Universidad Católica de la Santísima Concepción.

Facultad de Medicina. Campus San Andrés.

Alonso de Ribera 2850.

Concepción. Chile

Fonos: 041-2735427 - 041-2735406

Correo Electrónico: ctroncosop@ucsc.cl

Agradecimientos: A los estudiantes que participaron en las entrevistas y grupo focal, por su valioso tiempo y voluntad.

\section{BIBLIOGRAFÍA}

1. Llor B.; Abad M.; García M.; Nieto J. Ciencias psicosociales aplicadas a la salud. Editorial Interamericana McGraw-Hill. Madrid. España. 1995.
2. Enciclopedia de la Psicología. Volumen 1. Editorial Océano. Barcelona. España. 2002.

3. Fraisse P.; Diaget J. Psicofisiología de la conducta. Tratado de psicología experimental. Editorial Paidos. Buenos Aires. Argentina. 1996.

4. Osorio J.; Weisstaub G.; Castillo C. Desarrollo de la conducta alimentaria en la infancia y sus alteraciones. Rev Chil Nutr 2002; 29(3): 280-285.

5. Cervera P.; Clapés J.; Rigolfas R. Alimentación y dietoterapia. 4a Edición. Editorial McGraw - Hill Interamericana. Colombia. 2004.

6. Serra, L1.; Aranceta J. Nutrición y Salud Pública. Métodos, Bases Científicas y Aplicaciones. Editorial ELSEVIER, MASSON, S.A. Barcelona, España. 2006.

7. Valenzuela A. Obesidad. Editorial Mediterráneo. Santiago. Chile. 2002.

8. Busdiecker S., Castillo C., Salas I. Cambios en los hábitos de alimentación durante la infancia: una visión antropológica. Rev Chil Pediatr 2000; 71 (1): 5-11.

9. Organización Panamericana de la Salud. Estrategia Regional y Plan de Acción para un Enfoque Integrado Sobre la Prevención y Control de las Enfermedades Crónicas. [online] [citado agosto 2008]. Disponible en WWW: http://www.paho.org/ Spanish/AD/DPC/NC/reg-strat-cncds.pdf

10. MINSAL. Guía para Universidades Saludables y otras Instituciones de Educación Superior. 2006. [online] [citado marzo 2009]. Disponible WWW: http://www.inta.cl/materialEducativo/guia_universidades_saludables2006.pdf

11. MINSAL. II Encuesta de Calidad de Vida y Salud. Chile 2006. Informe de Resultados. Total Nacional. Subsecretaría de Salud Pública. División de Planificación Sanitaria. Departamento de Epidemiología. Unidad de Estudios y Vigilancia de Enfermedades No Transmisibles. [online] [citado agosto 2008]. Disponible WWW: www.epi.minsal.cl

12. Ministerio de Educación. Matrícula de Educación Superior. Matrícula Pregrado para los años 1990 a 2007. [online] [citado agosto 2008]. Disponible en WWW: http://compendio.educasup.cl/ docMatriculas/3.1_2007.xls

13. Sjostedt J.; Schumaker J., Nathawat S. Eating Disordens among Indian and Australian University Students. J Social Psychol 2001;138(3): 351-357.

14. Timko A., Perone J., Crossfield A. Are you currently on a diet? What respondents mean when they say "Yes". Eating Disorders 2006;14:157-166.

15. Von Bothmer M., Fridlundi Bengt F. Gender differences in healt habist and in motivación for a healthy 
lifestyle among Swedisch University sudents. Nursing Health Sciences 2005;7:107-118.

16. Unusan N. How university students evaluate the gramatical style of nutrition messages?. British Nutr Foundation 2006;31:100-103.

17. Arroyo M., Rocandio A., Ansotegui et al. Calidad de la dieta, sobrepeso y obesidad en estudiantes universitarios. Nutr Hospitalaria 2006;21(6): 673679.

18. Souza F., Martins M., Monteiro F et al. Anorexia e bulimia nervosa em alunas da Faculdade de Medicina da Universidade Federal do Ceara - UFC. Rev Psiquiatría Clín (Sao Paulo) 2002;2(4):172-180.

19. Oliveira A.; Mirada C.; Mendes M et al. Tobacco and health in the view of univerity students. Rev Brasileña Enfermería, (Brasilia) 2008;61(4):423427.

20. Vargas M.; Becerra F.; Prieto E. Evaluación antropométrica de estudiantes universitarios en Bogotá, Colombia. Rev Salud Pública 2008;10(3):433442.

21. Montero A.; Úbeda N. Gracia A. Evaluación de los hábitos alimentarios de una población de estudiantes universitarios en relación con sus conocimientos nutricionales. Rev Nutr Hospitalaria 2006;21(4):466473.

22. Martínez C.; Veiga P.; López A. et al. Evaluación del estado nutricional de un grupo de estudiantes universitarios mediante parámetros dietéticos y de composición corporal. Rev Nutr Hospitalaria 2005;20(3):197-203.

23. Olivares S.; Lera L.; Bustos N. Etapas del cambio, beneficios y barreras en actividad física y consumo de frutas y perdurasen estudiantes universitarios de Santiago de Chile. Rev Chil Nutr 2008;35(1):2535.

24. MacMillan N. Valoración de hábitos de alimentación, actividad física y condición nutricional en estudiantes de la Pontifica Universidad Católica de Valparaíso. Rev Chil Nutr 2007;34(4):330-336.

25. Mena M.; Faci M.; Ruch A. et al. Diferencias en los hábitos alimentarios y conocimientos, respecto a las características de una dieta equilibrada, en jóvenes con diferente índice de masa corporal. Rev Española Nutr Comunitaria 2002; 8(1):19-23.

26. Skemiene L.; Ustinaviciene R.; Piesine L. et al. Peculiarities of medical students' nutrition. Medicina (Kaunas) 2007;43(2):145-152.

27. Szewczynski JA.; Ostrowska A. Changes of dietary among Warsaw Medical University students in the years 1985-2001. Wiad Lek 2002;55(1):489-495.

28. Oliveras M.; Nieto P.; Agudo E. et al. Nutricional assessment of a university population. ORev Nutr Hospitalaria 2006;21 (2):179-183.

29. Cova F.; Alviar W.; Aro M. et al. Problemas de salud mental en estudiantes de la Universidad de Concepción. Rev Terapia Psicol 2007;25(2):105-112.

30. Ruiz A.; Morillo L. Epidemiología Clínica. Investigación Clínica Aplicada. Editorial Médica Panamericana. Primera Edición. Colombia. 2004.

31. Pineda E.; De Alvarado E. Metodología de la Investigación. Organización Panamericana de la Salud. 2008. Tercera Edición. Washington. Capítulo 8. pp. 102. 2008. 